Marquette University

e-Publications@Marquette

School of Dentistry Faculty Research and

Publications

Dentistry, School of

$12-2009$

\title{
Dental Treatment under General Anesthesia in Healthy and Medically Compromised/Developmentally Disabled Children: A Comparative Study
}

\author{
Juan Pablo Loyola-Rodriguez \\ San Luis Potosi University \\ Veronica Zavala-Alonso \\ San Luis Potosi University \\ Cinthya L. Gonzalez-Alvarez \\ San Luis Potosi University \\ Lilia A. Juarez-Lopez \\ National University of Mexico \\ Nuria Patiño-Marin \\ San Luis Potosi University
}

See next page for additional authors

Follow this and additional works at: https://epublications.marquette.edu/dentistry_fac

Part of the Dentistry Commons

\section{Recommended Citation}

Loyola-Rodriguez, Juan Pablo; Zavala-Alonso, Veronica; Gonzalez-Alvarez, Cinthya L.; Juarez-Lopez, Lilia A.; Patiño-Marin, Nuria; and Gonzalez, Cesar D., "Dental Treatment under General Anesthesia in Healthy and Medically Compromised/Developmentally Disabled Children: A Comparative Study" (2009). School of Dentistry Faculty Research and Publications. 247.

https://epublications.marquette.edu/dentistry_fac/247 


\section{Authors}

Juan Pablo Loyola-Rodriguez, Veronica Zavala-Alonso, Cinthya L. Gonzalez-Alvarez, Lilia A. Juarez-Lopez, Nuria Patiño-Marin, and Cesar D. Gonzalez 


\title{
Dental Treatment under General Anesthesia in Healthy and Medically Compromised/Developmentally Disabled Children: A Comparative Study
}

\author{
Juan Pablo Loyola-Rodriguez * / Veronica Zavala-Alonso ** / Cinthya L Gonzalez-Alvarez *** / \\ Lilia A Juarez-Lopez **** / Nuria Patiño-Marin ***** / Cesar D Gonzalez ******
}

\begin{abstract}
Aim: To compare the type, number of procedures and working time of dental treatment provided under dental general anesthesia (DGA) in healthy and medically compromised/developmentally disabled children (MCDD children). Design: This cross-sectional prospective study involved 80 children divided into two groups of 40 children each. Group 1 consisted of healthy and Group 2 consisted of MCDD children. Results: Healthy children needed more working time than MCDD children, the means being $161 \pm 7.9$ and $84 \pm 5.7$ minutes, respectively $(P=0.0001)$. Operative dentistry and endodontic treatments showed a significant statistical difference $(P=0.0001)$. The means of procedures were $17 \pm 5.0$ for healthy children and $11 \pm 4.8$ for MCDD children $(P=0.0001)$. Conclusions: Healthy children needed more extensive dental treatment than MCDD children under DGA. The information from this sample of Mexican children could be used as reference for determining trends both within a facility as well as in comparing facilities in cross-population studies.
\end{abstract}

Keywords: dental treatment, general anesthesia, outpatient anesthesia, oral rehabilitation J Clin Pediatr Dent 34(2): 177-182, 2009

\section{INTRODUCTION}

A lthough the overall prevalence of dental caries has declined in the world, there are still a substantial number of healthy children with early childhood

* Juan Pablo Loyola-Rodriguez, DDS, PhD, Head \& Chairman, The Master's Degree in Dental Science with specialization in Advanced General Dentistry Program at San Luis Potosi University, Mexico.

** Veronica Zavala-Alonso, DDS, MS, Assistant Professor, The Master's Degree in Dental Science with specialization in Advanced General Dentistry Program at San Luis Potosi University, Mexico.

*** Cinthya L. Gonzalez-Alvarez, DDS, 2nd year resident, The Master's Degree in Dental Science with specialization in Advanced General Dentistry Program at San Luis Potosi University, Mexico.

**** Lilia A. Juarez-Lopez, DDS, PhD, Associate Professor, Department of Pediatric Dentistry, National University of Mexico, Campus Iztacala, Mexico.

****** Nuria Patiño-Marin, DDS, PhD, Associate Professor, The Master's Degree in Dental Science with specialization in Advanced General Dentistry Program at San Luis Potosi University, Mexico.

****** Cesar D. Gonzalez, DDS, MS, Associate professor, Director Division of Pediatric Dentistry, Marquette University School of Dentistry, USA.

Send all coreespondence to: Juan Pablo Loyola-Rodriguez, Head \& Chairman, The Master's Degree in Dental Science with specialization in Advanced General Dentistry Program, San Luis Potosi University, Mariano Avila \# 295-2, Col. Tequisquiapam, CP 78250, San Luis Potosí, SLP, México.

Tel: 524448262361 ext. 102

Fax: 524448262361 ext. 104

E-mail: jloyola@uaslp.mx caries who display uncooperative behaviors of such magnitude that oral rehabilitation under dental general anesthesia (DGA) is required, especially in developing countries. ${ }^{1}$ The proportion of medically compromised children and those with developmental disabilities who survive is increasing and these children remain at high risk of dental caries and periodontal disease. ${ }^{2}$ Therefore, there is an increasing need for comprehensive dental care for both non-cooperative healthy children and medically compromised children that will require special procedures and techniques in the delivery of dental treatment.

A special concern is the cost of medical and dental care of disabled people. One report showed that expenses in health care for the handicapped population, especially those affected with developmental disabilities, is approximately $\$$ 4.7 billion (US dollar) per year without including costs for dental treatment. ${ }^{3}$ In several countries, use of DGA as an alternative to provide dental treatment is quite expensive and available national health insurances, both public and private, do not cover its costs. Worst of all, many families with disabled members have a low income, making it difficult to afford any medical and/or dental treatment. ${ }^{4}$ Besides, it has been reported that DGA is used as multidisciplinary approach for oral rehabilitations under DGA that involve personnel with different dental specialities which increase the procedure cost. ${ }^{4-6}$

The availability of rapid and short-acting anesthetics such as sevoflurane and propofol has improved the general anesthesia (GA) technique and facilitated the early recovery of 
patients after ambulatory (outpatient) general anesthesia. ${ }^{6}$ The use of propofol as an intravenous induction agent is very useful, having been associated with fast clearance and reducing postoperative nausea and vomiting. ${ }^{7}$ All the above mentioned advancements have significantly improved the safety of anesthetic technique, a reduction in adverse events, and has resulted in increased patient satisfaction with DGA in an office-based ambulatory setting. ${ }^{\text {? }}$

It has been reported that persons with disabilities consistently show poor oral hygiene, a high level of periodontal disease and dental caries. ${ }^{8,9}$ However, it is important to note that most data are from descriptive studies where there is no standardization of criteria, which could influence results. On the other hand, it is important to take into account the type of disability, age and demographic situation of each population studied. ${ }^{10}$ The aim of this investigation was to determine and compare the type, number of procedures and working time length of dental treatment provided under dental general anesthesia in healthy and in medically compromised/developmentally disabled (MCDD) children.

\section{MATERIAL AND METHODS}

The Clinical Studies Committee of the Master's Degree Program in Dental Science with Specialization in Advanced General Dentistry of the Faculty of Dentistry at San Luis Potosi University, México, approved this study. Parents of children completed a standardized health questionnaire that included information about pediatric and oral evaluations, and the last course of antibiotics. An informed and voluntary written consent from parents was obtained prior to clinical examinations according to the ethical principles of the world medical association declaration of Helsinki (version 2002). This cross-sectional prospective study involved 80 subjects divided into two groups of 40 children each. Group 1 consisted of healthy children and Group 2 consisted of MCDD children. All children in the study had no previous dental treatment and exhibited negative or definitively negative behavior on Frankl's scale. ${ }^{11}$ Attendant risks and benefits of general anesthesia and dental treatment required were explained and written consent obtained from parents or guardians.

A non-probabilistic consecutive sampling was used from January 2000 through December 2006 in two ambulatory outpatient care facilities. Patients were recruited at the Faculty of Dentistry at San Luis Potosi University and the Faculty of Dentistry of The National University of Mexico, Campus Zaragoza. The inclusion criteria were as follows: 1) clinical diagnosis of negative or definitively negative behavior as defined by Frankl, ${ }^{11}$ 2) no previous dental treatment or dental care, 3) age of patient between 2 and 7 years old, and 4) patients classified as American Society of Anesthesiology $^{12}$ (ASA) I (Group 1) or II/III (Group 2). The exclusion criteria consisted of patients with a history of difficulty in tracheal intubation and patients that did not consent to be treated. All patients that participated in this study fulfilled the inclusion criteria; all variables included were blinded analyzed in both institutions.
Oral, medical examinations and development of treatment plans

The same pediatric dentist at each institution performed a dental history, an oral exam and when possible a radiographic examination. In most cases, radiographic examinations were carried out during the DGA procedure due to the impossibility to do so during the medical and dental examination. Similarly, the same pediatric dentist at each institution developed all treatment plans. Parents were informed but did not participate in the development of the treatment plan. Both pediatric dentists were calibrated regarding diagnosis and treatment planning through a pilot study that included 5 patients, and the kappa statistic between both pediatric dentists was 0.8-1.0. A pediatrician and an anesthesiologist in each institution carried out a medical clinical history and pre anesthetic evaluation. The multidisciplinary team that participated in the entire process included anesthesiologists, a dental assistant, pediatrician, nurse, pediatric dentist, and 2nd year residents from the Advanced General Dentistry Program (AGDP). All procedures regarding behavior guidance and pre-anesthetic evaluation in both institutions were carried out according to the American Academy of Pediatric Dentistry Guidelines. ${ }^{13-15}$

All patients received inhaled sevoflurane (Abbott Lab., Bogotá, Colombia) anesthesia through facial mask induction carried out according to a standard protocol. Balanced maintenance anesthesia was used in all cases using intravenous (IV) propofol (Recofol, $200 \mathrm{mg} / 20 \mathrm{ml}, 2-3 \mathrm{mg} / \mathrm{kg}$; Lab. Pisa, Lieras Oy, Turku, Finland); Tracrium (atracurium, 10 $\mathrm{mg} / \mathrm{ml}, 0.3-0.6 \mathrm{mg} / \mathrm{kg}$, Glaxo Wellcome Foundation Lab, London, UK) as the neuromuscular blocker; Sevoflurane (Abbott Lab., Bogotá, Colombia) as the inhalatory anesthetic; Ketorolac $(30 \mathrm{mg} / \mathrm{ml}, 1 \mathrm{mg} / \mathrm{kg}$, Lab. Pisa, Guadalajara, Mexico) as an analgesic, as we described previously. ${ }^{16}$ Local anesthetic (Lidocaine HCL and epinephrine 2\%, Laboratorios Zeyco, Zapopan, Jal, Mexico) was used for pain management and hemorrhage control in cases of oral surgery and endodontic treatment. All oral rehabilitations were completed with the patient in a dental chair (Adec Performer, Portland, OR, USA). Clinical diagnostic and treatment planning at both institutions were in accordance with the guidelines of dental treatment for children of San Luis Potosi University.

\section{Dental treatment under general anesthesia}

The same pediatric dentist at each institution performed all dental procedures for all children participating in the study. All restorative procedures completed during DGA for both groups were in accordance with the American Academy of Pediatric Dentistry Guidelines. ${ }^{17-19}$

A Welch Allyn Atlas Monitor equipped with Electrocardiogram (model 622SO, Skaneateles Falls, NY, USA) and anesthetic machine (Narkomed 2A11, North American Drager, PA, USA) were used to monitor anesthesia care in each outpatient setting. Heart rate, temperature, arterial hemoglobin oxygen saturation, and arterial pressure were recorded and monitored throughout the procedures and patient recovery. 
Patients were discharged when they were fully awake and met Aldrete's criteria for patient discharge ${ }^{20}$ and after appropriate written instructions were reviewed. Follow-up appointments for the next day and a week later were programmed for all patients.

Data collected for statistical analysis included the type of dental treatment provided, the number of procedures completed per patient, and the length of time under DGA, including both the length of time to complete the dental procedures and the total time from induction to recovery. ${ }^{21}$

\section{Statistical analyses}

To summarize and compare data between groups, we used a novel classification that includes an ordinal scale to measure the type of procedures completed, the number of procedures completed, and length of time required to complete these procedures while the patient was under DGA. We used JMP program version 4.0 (SAS Institute) for statistical analysis, statistical significance was set at $P<0.05$. ShapiroWilks, Levene and Brown Forsythe tests were used to test the normality of distribution of all variables. The non-parametric Mann Whitney U test was used to compare continuous variables; Fisher's exact and $\mathrm{X}^{2}$ of Mantel-Haenszel tests were used to compare categorical variables. Analysis of covariance (ANCOVA) was performed to control the influence of confounding variables.

\section{RESULTS}

Distribution according to gender and the relationship of age and gender with $D G A$

Total sample included fifty-one males (64\%) and twentynine females $(37 \%)$. Healthy children were classified in an age range of 2-6 years old, the mean being 50 months (4 years and 2 months). In MCDD children the distribution was in a higher range of age (2-7 years old), the mean being 65 months (5 years and 5 months). An interesting point to note was that males were more frequently assigned to DGA than females in both groups, twenty-seven in group 1 and twentyfour in group 2. When age and gender were compared between groups, there was no statistical difference $(P>$ $0.05)$.

The healthy children were classified as ASA I and MCDD children were classified as medically compromised (ASA II-III) in accordance with the classification of physical status of The American Society of Anesthesiology. The healthy children were classified as definitely negative (level I) and MCDD children were classified as definitely negative and negative (levels I and II, respectively) in accordance with Frankl's classification. ${ }^{11}$ When both variables ASA classification and scale for behavior were compared between groups, there was a significant statistical difference $(P=$ $0.0001)$.

\section{Diagnostic findings of the medically compromised children}

The relationship between medical diagnosis and gender is shown in Table 1. Patients with cognitive delays and neuromuscular disorders received dental treatment under DGA
Table 1. Clinical diagnoses of MCDD children that received dental treatment under DGA in relation to gender

\begin{tabular}{lcccc}
\hline Clinical diagnostic & M & F & Total & $\%$ \\
\hline Asthma & 2 & 0 & 2 & 5 \\
\hline Autism & 1 & 0 & 1 & 2.5 \\
\hline Seizures disorders & 0 & 1 & 1 & 2.5 \\
\hline Ehlers Danlos syndrome & 1 & 0 & 1 & 2.5 \\
\hline Breath-holding spells & 0 & 1 & 1 & 2.5 \\
\hline Cerebral palsy & 4 & 9 & 13 & 32.5 \\
\hline Hydrocephalus & 2 & 0 & 2 & 5 \\
\hline Intellectual disability & 7 & 2 & 9 & 22.5 \\
\hline Deafness & 0 & 1 & 1 & 2.5 \\
\hline Down syndrome & 6 & 1 & 7 & 17.5 \\
\hline GSNI & 1 & 1 & 2 & 5 \\
\hline Total & 24 & 16 & 40 & 100 \\
\hline
\end{tabular}

MCDD medically compromised/developmentally disabled, DGA (dental general anesthesia); M (male); F (female); GSNI (genetic syndrome not identified).

most frequently (55\%). The following disorders were found most often in the affected population: cerebral palsy (32.5\%), intellectual disability $(22.5 \%)$, and the genetic disorder Down syndrome (17.5\%).

\section{General anesthesia time, working time length and discharge time}

The results relating total GA time, from induction to recovery, in both groups are shown in Table 2. There was a significant statistical difference of $(P=0.0001)$ between the mean minutes for healthy children group $(185 \pm 8.9)$ and for MCDD group (94 \pm 5.7$)$. Healthy children needed more actual procedure time than MCDD children, with a significant statistical difference $(P=0.0001)$ between the means $161 \pm 7.9$ for healthy children and $84 \pm 5.7$ for MCDD children, respectively. There was no statistical difference in discharge time, $11 \pm 3$ for healthy children and $12 \pm 3$ for MCDD children.

Table 2. General anesthesia time, working time length and discharge time between groups

\begin{tabular}{lcccccc}
\hline & \multicolumn{2}{c}{ GAT } & \multicolumn{2}{c}{ WTL } & \multicolumn{2}{c}{ DT } \\
\hline & Mean \pm SD & Range & Mean \pm SD & Range & Mean \pm SD & Range \\
\hline Healthy & $185 \pm 8.9$ & $35-255$ & $161 \pm 7.9$ & $25-225$ & $11 \pm 3$ & $1-15$ \\
\hline MCDD & $94 \pm 5.7$ & $28-166$ & $84 \pm 5.7$ & $18-156$ & $12 \pm 3$ & $5-18$ \\
\hline
\end{tabular}

All data are expressed in minutes. GAT (general anesthesia time); WTL (working time length); DT (discharge time); SD (standard deviation); MCDD (medically compromised/developmentally disabled)

\section{Working time length and type of procedures}

Table 3 shows that $80 \%$ of oral rehabilitations for healthy children had a working time of $>120$ minutes $(E)$, while the MCDD showed that $67.5 \%$ of children needed $<120$ minutes $(\mathrm{S}, \mathrm{M}, \mathrm{L})$. This is a statistically significant difference $(P=$ 0.001). Results related to type of dental treatment showed that $85 \%$ of healthy children needed operative dentistry and endodontic treatment (T4) in the primary dentition without oral surgery; but $57 \%$ of MCDD children group received 
Table 3. Working time length and type of dental procedures under DGA

\begin{tabular}{lccccccccc}
\hline & \multicolumn{4}{c}{ Working time length } & \multicolumn{6}{c}{ Type of procedure } \\
& S & $\mathrm{M}$ & $\mathrm{L}$ & $\mathrm{E}$ & $\mathrm{T} 1$ & $\mathrm{~T} 2$ & $\mathrm{~T} 3$ & $\mathrm{~T} 4$ & $\mathrm{~T}$ \\
\hline Healthy & & & & & & & & & \\
Frequency & 2 & 1 & 5 & 32 & 0 & 1 & 4 & 34 & 1 \\
\hline$\%$ & 5 & 2.5 & 12.5 & 80 & 0 & 2.5 & 10 & 85 & 2.5 \\
\hline
\end{tabular}

MCDD

$\begin{array}{llllllllll}\text { Frequency } & 5 & 13 & 9 & 13 & 7 & 5 & 14 & 10 & 4\end{array}$

\begin{tabular}{llllllllll}
\hline$\%$ & 12.5 & 32.5 & 22.5 & 32.5 & 17.5 & 12.5 & 35 & 25 & 10
\end{tabular}

DGA (dental general anesthesia), MCDD (medically compromised/developmentally disabled; S (short); M (medium); L (long), E (extended); T1 (type 1), T2 (type 2); T3 (type 3); T4 (type 4); T5 (type 5) in accordance with the classification of treatment under DGA.

oral surgical services (T1, T2 and T5). When the type of dental procedure between groups was compared, there was a significant statistical difference $(P=0.0001)$. Healthy children received a mean of $17 \pm 5.0$ treatment procedures while children in the MCDD group received a mean of $11 \pm 4.8$ treatment procedures, which was statistically significant $(P=0.0001)$.

\section{Operative dentistry, oral surgery and endodontic treatments}

Table 4 shows that the total number of operative dentistry treatment procedures carried out in healthy children were 475 and 275 in the MCDD children. A similar tendency was found in endodontic treatment showing that 123 root canal treatments were carried out in healthy children but only 58 endodontic procedures in MCDD children. There was a significant statistical difference when these two variables were compared between groups, $(P=0.0001)$. There was no statistical difference $(P>0.05)$ between groups in the number of oral surgery procedures.

Table 4. Operative dentistry, oral surgery and endodontic treatment in children carried out under DGA.

\begin{tabular}{lllllll} 
Procedure & Frequency & Mean & Range & Frequency & Mean & Range \\
\hline $\begin{array}{l}\text { Operative } \\
\text { dentistry }\end{array}$ & $475^{*}$ & 11.6 & $1-15$ & $275^{*}$ & 6.8 & $1-13$ \\
\hline $\begin{array}{l}\text { Oral } \\
\text { surgery } 92\end{array}$ & 2.4 & $1-8$ & 99 & 2.8 & $1-20$ \\
\hline $\begin{array}{l}\text { Endodontic } \\
\text { treatment }\end{array} 123^{*}$ & 3.0 & $1-12$ & $58^{*}$ & 1.4 & $1-3$ \\
\hline
\end{tabular}

DGA (dental general an anesthesia), MCDD (medically compromised/developmentally disabled. ('Significant statistal difference)

\section{Comparative data of most important variables}

The most important variables that showed statistical difference when both groups were compared are shown in Table 5. Healthy children had a mean age younger than that of patients of MCDD at time of treatment delivery and males needed DGA more frequently. The number of dental procedures under DGA had a direct impact on total general anesthesia time and working time length. The type of treatment
Table 5. Comparative data of most important variables between groups

\begin{tabular}{llll}
\hline & Healthy & MCDD & $p$ value \\
\hline WTL & $161 \mathrm{~min}$ & $84 \mathrm{~min}$ & 0.0001 \\
\hline GAT & $185 \mathrm{~min}$ & $94 \mathrm{~min}$ & 0.0001 \\
\hline Behaviour & $\mathrm{I}$ & $\mathrm{I}, \mathrm{II}$ & 0.0001 \\
\hline Dental procedures & 17 & 11 & 0.0001 \\
\hline Operative dentistry & 475 & 275 & 0.0001 \\
\hline Endodontic treatment & 123 & 58 & 0.0001 \\
\hline Type of procedure & $\mathrm{E}$ & $\mathrm{S}, \mathrm{M}, \mathrm{L}$ & 0.0001 \\
\hline Procedures level & $\mathrm{L} 5$ & $\mathrm{~L} 1, \mathrm{~L} 2, \mathrm{L3}, \mathrm{L} 4$ & 0.0001 \\
\hline
\end{tabular}

Min. (minutes); MCDD (medically compromised/developmentally disabled); WTL (working time length), GAT (general anesthesia time); behavior was classified in accordance with Frankl's classification. E (extended); S (short), M (medium), L (long), $\mathrm{L}$ (level) all according to the classification of dental treatment under dental general anesthesia.

carried out included more operative dentistry and root canal treatment for healthy children. The number of procedures was quite different with healthy children showing a greater necessity of dental treatment than MCDD children.

The ANCOVA test was applied to control the confounding variables of group, age, working time and type of dental procedures in relation to number of dental procedures carried out under DGA. Working time was the only variable that showed a significant statistical difference $(P=0.0001)$.

\section{DISCUSSION}

Dental treatment provided under DGA in different settings is important for a particular group of patients, such as noncooperative healthy children and MCDD children, adolescents and adults. It has been reported that persons with disabilities consistently show poor oral hygiene, high level of periodontal disease and dental caries. ${ }^{8,9}$ These descriptive studies involved children with different disabilities, age and demographic situations without standardization of criteria, which can influence results.

The present study showed differences in age distribution, the mean age was 4 years and 2 months for the healthy children while MCDD children showed a mean age of 5 years and 5 months at the time of treatment. This finding agrees with a report in which patients with special needs were older when treated than non-special needs patients. ${ }^{22}$ This could be explained due that most patients come from low-income families that lived in small communities in several states of the central area of Mexico with difficulties for transportation to DGA setting; this situation produce a delay of information, waiting time and economical difficulties to deliver DGA procedures. Besides, most MCDD children have complicated health needs with high priority for the family as a result produces a later referral for dental treatment.

The fact that in this study we had more males than females in our sample is consistent with previous studies, ${ }^{23,24}$ but in contrast to results from others. ${ }^{25-27}$ There is not a clear explanation for this difference in gender distribution, but it has been suggested that a higher prevalence of neuropsychiatric disorders among boys could explain the predominance of males. ${ }^{22}$

It has been reported that in hospital settings, the 
frequency of DGA is concentrated in patients affected with cardiac problems, asthma, physical and emotional disabilities. ${ }^{28}$ However, in a study carried out in Spain it was reported that the main frequency of oral rehabilitations under DGA were in intellectual disability, cerebral palsy, epilepsy, and Down syndrome patients. ${ }^{29}$ This frequency is quite similar to this outpatient DGA study, suggesting that demographic, ethnic and treatment facilities play a major role in each study. There are reports describing dental treatment needs of healthy children (ASA I), but there are few studies comparing dental treatment necessities between healthy and MCDD children. One study shows that ASA I and II children needed more dental treatment under DGA than ASA III and IV children. ${ }^{30}$ However, because ASA I children required more dental treatment than ASA II children, we considered it important to separate healthy and MCDD populations when comparative studies are undertaken. In the present study, healthy children seeking dental care under DGA had greater treatment needs than MCDD children. These findings are in contrast with a previous report where MCDD children required more time to complete dental treatment under general anesthesia. ${ }^{9}$ However, the present results are similar to a recent publication in which patients with special needs had fewer teeth treated than non-special needs patients and that males in both groups needed more dental treatment than females. ${ }^{22}$

The information about the time of GA in operative setting room is sparse. ${ }^{21}$ In the present study we found that healthy children required more general anesthesia time for their oral rehabilitation. The mean was 185 minutes for healthy and 94 minutes for MCDD children, which was a statistically significant difference $(P=0.0001)$. Another important point in planning DGA is to estimate the working time, especially to consider the cost of the intervention. We found a mean of 161 and 84 minutes for healthy and MCDD children, respectively. Unfortunately, we could not compare these variables with other studies due to variability in study design and room settings. Recovery time was similar for both groups, means being 11 and 12 minutes for each group respectively. This finding is similar to several reports of general anesthesia procedures in different medical specialties where the combination propofol-sevoflurane is used for GA..$^{31}$

The results of this study showed that $80 \%$ of the total sample of healthy children required greater than 120 minutes to complete the dental procedures, whereas most $(67.5 \%)$ of the MCDD children required less than 120 minutes of DGA. It is important to note that there was not a statistical difference when comparing the number of teeth extracted in both groups. Therefore, eliminating the possibility that the longer working time in Group I was due to more teeth being restored as opposed to being extracted. These findings are in contrast with other studies that report that MCDD children required more time to complete the dental treatment. ${ }^{9}$ A possible explanation could be that healthy children have greater access to a more cariogenic diet than MCDD children through their parents or relatives. It is also possible that other factors such as oral hygiene habits and genetic susceptibility to dental caries play a role in this process. An increase in risks of dental diseases for MCDD children also exists due to the difficulties in having access to dental health care and the burden of additional cost of dental treatment. ${ }^{26}$ However, countering these difficulties is the increase in the availability of information about oral health and parents and/or guardians having more knowledge about oral hygiene and the need for preventive dental treatment.

Because the type and number of procedures are difficult to compare or analyze with other studies, the authors developed a classification system based on three main factors: working time length, type and number of procedures carried out under DGA which attempts to conform with a more multidisciplinary approach to the delivery of care. ${ }^{21}$ Since then, we have used this classification to describe the entire process of dental care under DGA rather than individual procedures and to organize and summarize data. There are some important points to note in this study. First the group of healthy children had a mean of 17 dental procedures while the MCDD group had a mean of 11 dental procedures carried out under DGA, with this difference being statistically significant. Operative dentistry, root canal treatments and stainless steel crowns, especially in the maxillary arch, were more often delivered in patients ASA I. This study agrees with the reports indicating that the number of dental procedures such as pulpotomies and stainless steel crowns are placed more often in healthy children than MCDD children. ${ }^{28}$

Currently, outpatient anesthesia is increasing in all medical fields due to the reductions in costs related to the elimination of extended hospital stays. This reduction in medical expenses enables lower income families a better opportunity to obtain safe, comprehensive preventive and restorative care. The approach to dealing with patients with special health care needs is changing, it has been incorporated a multidisciplinary team approach concept that delivers a wide range of dental treatment possibilities and helping to provide a better quality of life for these patients. The combination propofol-sevoflurane is an anesthetic technique with a very good cost/benefit ratio and has an excellent recovery time with a reduction in vomiting and nausea as postoperative complications.

Patients with special health care needs often require oral rehabilitation under DGA in the hospital setting and worldwide; many available insurance plans do not include this as a covered benefit. It has been reported that office-based DGA is a safe procedure with a high level of patient satisfaction. ${ }^{32,33}$ It is therefore possible that DGA provided in an outpatient dental clinic or office setting may increase in frequency in the future, but funding will come primarily from the patient or family financial resources. In this regard, especially in developing countries, public institutions such as dental schools will play an important role in providing dental treatment for people with disabilities, and healthy children affected by early childhood caries. It is important to mention that all children in this study received follow-up care that included oral hygiene instructions, fluoride application and diet education.

We concluded that healthy children needed more working 
time and number of dental procedures, especially in operative dentistry and endodontic treatment, than MCDD children under DGA. The information from this sample of Mexican children could be used as reference for determining trends both within a facility as well as in comparing facilities in cross-population studies.

\section{ACKNOWLEDGMENTS}

UASLP-FAI supported this study. The authors thank Dr. Brian Hodgson for his technical assistance with English.

\section{REFERENCES}

1. Loyola-Rodríguez JP, Aguilera-Morelos AA, Torres-Juárez A, SantosDíaz MA, González-Azúara K. Dental treatment under general anesthesia of children with special needs. Bol Med Hosp Infant Mex, 59: 288-296, 2002.

2. Ghezzi EM, Chávez E, Ship JA. General anesthesia protocol for the dental patient: emphasis for older adults. Spec Care Dentist, 20: 81-108, 2000.

3. Waldman HB \& Perlman SP. Dental Care for individuals with developmental disabilities is expensive, but needed. J Calif Dent Assoc, 30: 427-432, 2002.

4. Jameson K, Averley PA, Shackley P, Steele J. A comparison of the 'cost per child treated' at a primary care-based sedation referral service, compared to a general anaesthetic in hospital. Br Dent J, 203: E13, 2007.

5. Maestre C. The use of general anaesthesia for tooth extraction in young handicapped adults in France. Br Dent J, 180: 297-302, 1996.

6. Manley MCG, Skelly AM, Hamilton AG. Dental treatment for people with challenging behaviour: general anaesthesia or sedation? $\mathrm{Br}$ Dent J, 188: 358-360, 2000.

7. Coyle TT, Helfrick JF, Gonzalez ML, Andresen RV, Perrott DH. Officebased ambulatory anesthesia: Factors that influence patient's satisfaction or dissatisfaction with deep sedation/general anesthesia. J Oral Maxill of Surg, 63: 163-172, 2005.

8. Shyama M, Al-Mutawa SA, Morris RE, Sugathan T, Honkala E. Dental caries experience of disabled children and young adults in Kuwait. Community Dent Health, 18: 181-186, 2001.

9. Mitsea AG, Karidis AG, Donta-Bakoyianni C, Spyropoulos ND. Oral health status in Greek children and teenagers with disabilities. J Clin Pediatr Dent, 26: 111-118, 2001.

10. Hulland S, Sigal MJ. Hospital-based dental care for persons with disabilities: a study of patient selection criteria. Spec Care Dentist, 20: 131-138, 2000.

11. Frankl SN, Shiere FR, Fogels HR. Should the parent remain in the operatory? J Dent Child, 29: 150-163, 1962.

12 Keats AS. The ASA classification of physical status: a recapitulation. Anesthesiology, 49: 233-236, 1978.

13. American Academy of Pediatric Dentistry: Guideline on behavior guidance for the pediatric dental patient. Pediatr Dent, 27: 92-100, 2005-2006

14. American Academy of Pediatric Dentistry: Guideline on use of anesthesia care providers in the administration of in-office deep sedation/general anesthesia to the pediatric dental patient. Pediatr Dent, 27: 119-121, 2005-2006
15. American Academy of Pediatric Dentistry: Clinical guideline on use of anesthesia-trained personnel in the provision of general anesthesia/deep sedation to the pediatric dental patient. Pediatr Dent, 26: 104-105, 2004.

16. Loyola-Rodriguez JP, Aguilera-Morelos AA, Santos-Diaz MA, ZavalaAlonso V, Davila-Perez C, Olvera-Delgado H, Patiño-Marin N, De Leon-Cobian I. Oral rehabilitation under general anesthesia, conscious sedation, and conventional techniques in patients affected by cerebral palsy. J Clin Pediatr Dent, 28: 279-284, 2004.

17. American Academy of Pediatric Dentistry: Clinical guideline on pediatric restorative dentistry. Pediatr Dent, 26: 106-114, 2004.

18. American Academy of Pediatric Dentistry: Guideline on pulp therapy for primary and young permanent teeth. Pediatr Dent, 27: 130-134, 2005-2006.

19. American Academy of Pediatric Dentistry: Guideline on pediatric oral surgery. Pediatr Dent, 27: 158-164, 2005-2006.

20. Aldrete JA, Kroulik D. Postanesthetic recovery score. Anesth Analg, 49: 924-934, 1970.

21. Loyola-Rodriguez JP, Zavala-Alonso NV, Patiño-Marin N, Friedman C. A new classification system for dental treatment under general anesthesia. Spec Care Dentist, 26: 25-29, 2006.

22. Haubek D, Fugisang M, Poulsen S, Rolling I. Dental treatment of children referred to general anaesthesia-association with country of origin and medical status. Int J Paediatric Dent, 16: 239-46, 2006.

23. Vermeulen M, Vinckier F, Vandenbroucke J. Dental general anesthesia: Clinical characteristics of 933 patients. J Dent Child, 58: 27-30, 1991.

24. Jamjoom MM, al-Malik MI, Holt RD, el-Nassry A. Dental treatment under general anaesthesia at a hospital in Jeddah, Saudi Arabia. Int J Paediatric Dent, 11: 110-116, 2001.

25. Messieha Z. Risks of general anesthesia for the special needs dental patient. Spec Care Dentist, 29: 21-25, 2009.

26. Atan S, Ashley P, Gilthorpe MS, Scheer B, Mason C, Roberts G. Morbidity following dental treatment of children under intubation general anaesthesia in a day-stay unit. Int J Paediatric Dent, 14: 9-16, 2004.

27. Vinckier F, Gizani S, Declerck D. Comprehensive dental care for children with rampant caries under general anaesthesia. Int J Paediatric Dent, 11: 25-32, 2001.

28. Ibricevic H., Al-Jame Q., Honkala S. Pediatric dental procedures under general anesthesia at the Amiri hospital in Kuwait. J Clin Pediatr Dent, 25: 337-342, 2001.

29. Rodríguez-Vázquez C, Garcillan MR, Rioboo R, Bratos E. Prevalence of dental caries in an adult population with mental disabilities in Spain. Spec Care Dentist, 22: 65-69, 2002.

30. Camilleri A, Roberts G, Ashley P, Scheer B. Analysis of paediatric dental care provided under general anaesthesia and levels of dental disease in two hospitals. Br Dent J, 196: 219-223, 2004.

31. Kataria B, Epstein R, Bailey A, et al. A comparison of sevoflurane to halothane in paediatric surgical patients: results of a multicentre international study. Paediatr Anaesth, 6: 283-292, 1996.

32. Maurer SM, Boggs AM, Mourino AP, Farrington FH. Recall intervals: effect on treatment needs of the handicapped patient: a retrospective study. J Clin Pediatr Dent, 20: 123-6, 1996.

33. Perrott DH, Yuen JP, Andresen RV, Dodson TB. Office-based ambulatory anesthesia: outcomes of clinical practice of oral and maxillofacial surgeons. J Oral Maxillof Surg, 61: 983-995, 2003. 